\title{
Sobre o gênero Phoroncidia: nova espécie, ecologia e descrição do macho de $P$. reimoseri com novas ocorrências para o sul do Brasil (Araneae, Theridiidae)
}

\author{
Everton Nei Lopes Rodrigues ${ }^{1,2}$ \& Maria Aparecida L. Marques ${ }^{2}$
}

1. Programa de Pós-Graduação em Biologia Animal, Departamento de Zoologia, Instituto de Biociências, Universidade Federal do Rio Grande do Sul, Av. Bento Gonçalves, 9500, Bloco IV, Prédio 43435, 91501-970 Porto Alegre, RS, Brasil. (enlrodrigues@yahoo.com.br) 2. Museu de Ciências Naturais, Fundação Zoobotânica do Rio Grande do Sul, Rua Dr. Salvador França, 1427, 90690-000 Porto Alegre, RS, Brasil. (maria-marques@fzb.rs.gov.br)

\begin{abstract}
On the genus Phoroncidia: new species, ecology and description of the male of P. reimoseri and new records for southern Brazil (Araneae, Theridiidae). Phoroncidia piratini sp. nov. from state of Rio Grande do Sul is described and illustrated, based on males and females. The male of $P$. reimoseri Levi, 1964 is described and illustrated for the first time and new records are provided. Ecological data are presented for both species.
\end{abstract}

KEYWORDS. Neotropical, spider taxonomy, riparian forest.

RESUMO. Phoroncidia piratini sp. nov. do estado do Rio Grande do Sul é descrita e ilustrada, com base em espécimes de ambos os sexos. O macho de P. reimoseri Levi, 1964 é descrito e ilustrado pela primeira vez e novos registros são fornecidos. Dados ecológicos de ambas as espécies são apresentados.

PALAVRAS-CHAVE. Neotropical, taxonomia de aranhas, mata ciliar.

O gênero Phoroncidia Westwood, 1835 é de ampla distribuição mundial, com exceção do norte da Europa e oeste da América do Norte (Levi \& Levi, 1962). Caracterizase pelo colulo constituído de duas cerdas, comumente escondidas sob um anel muito esclerotinizado que circunda as fiandeiras, carapaça projetada anteriormente na região ocular, abdome de várias formas, amplamente esclerotinizado, frequentemente coriáceo, lateralmente pregueado, com tubérculos ou projeções que podem variar em número e tamanho (LeVI \& Levi, 1962; LevI, 1964). Poucos estudos trataram do comportamento e ecologia de aranhas do gênero (Marples, 1955; EBERHARD, 1981).

Atualmente o gênero consta com 74 espécies, sendo a espécie-tipo Phoroncidia aculeata Westwood, 1835 descrita para a Índia; para a Região Neotropical são conhecidas 25 espécies (Platnick, 2010). Para o Brasil são oito espécies, $P$. biocellata (Simon, 1893) para o estado de Minas Gerais, P. longiceps (Keyserling, 1886) para o Pará, P. moyobamba Levi, 1964 para os estados de Roraima e Amazonas, P. reimoseri Levi, 1964 para São Paulo, Santa Catarina e Rio Grande do Sul, $P$. rubromaculata (Keyserling, 1886) para o Rio de Janeiro, P. studo Levi, 1964 para o Amazonas, P. tina Levi, 1964 para Minas Gerais e P. tricuspidata (Blackwall, 1863) para o estado do Rio de Janeiro (Levi, 1964; Buckup \& Marques, 1989; Platnick, 2010).

Neste trabalho é descrita uma nova espécie do estado do Rio Grande do Sul, representada por ambos os sexos e o macho de Phoroncidia reimoseri, é descrito, ilustrado e registrado pela primeira vez para o estado do Paraná e são apresentadas novas ocorrências para Santa Catarina e Rio Grande do Sul, além de dados ecológicos de ambas as espécies coletadas em matas ciliares no Rio Grande do Sul.

\section{MATERIAL E MÉTODOS}

Taxonomia. Os espécimes estudados estão depositados na coleção de aranhas do Museu de Ciências Naturais, Fundação Zoobotânica do Rio Grande do Sul (MCN, Erica H. Buckup) em Porto Alegre, Rio Grande do Sul, Brasil. As descrições e terminologia das estruturas da genitália de ambos os sexos seguem Levi (1964) e Agnarsson et al. (2007). A genitália da fêmea foi clarificada com ácido lático $85 \%$ por aproximadamente 30 minutos, em temperatura ambiente, até a perfeita visualização das estruturas internas. Para a expansão do palpo, a estrutura foi submersa em $\mathrm{KOH} 10 \%$ por aproximadamente 24 horas e depois imersa em água destilada. Todas as medidas são apresentadas em milímetros. Abreviaturas: OMA, olhos médios anteriores; OMP, olhos médios posteriores; OLA, olhos laterais anteriores e OLP, olhos laterais posteriores.

Áreas de estudo. Foram realizadas coletas em quatro matas ciliares no estado do Rio Grande do Sul, Brasil. Na mata ciliar do rio Piratini, no baixo curso, município de Arroio Grande na região sul da Planície Costeira ( $\left.31^{\circ} 54^{\prime} 06.47^{\prime \prime} \mathrm{S}, 52^{\circ} 39^{\prime} 08.29^{\prime \prime} \mathrm{W}\right)$. O trecho selecionado está aproximadamente $14 \mathrm{~m}$ acima do nível do mar e $39 \mathrm{~km}$ de extensão ao longo do vale fluvial, possui 4000,45 ha de mata ciliar, representando o maior segmento florestal contínuo nativo inserido atualmente na região sul da Planície Costeira. Na mata ciliar do rio Camaquã, na margem esquerda do baixo curso, no município de Cristal ( $\left.31^{\circ} 01^{\prime} 01.70 ” S, 51^{\circ} 56^{\prime} 42.01^{\prime \prime W}\right)$, na 
região centro-sul da Planície Costeira. Aproximadamente $14 \mathrm{~m}$ acima do nível do mar. A área encontra-se em bom estado de conservação, a mata é contínua, sobre uma área plana com poucos desníveis. O local sofre influência de enchentes, determinando poucos indícios de perturbação antrópica. Mata ciliar do rio dos Sinos, no município de Parobé, margem direita do rio (2941'06.94"S, $\left.50^{\circ} 51^{\prime} 05.98^{\prime \prime} \mathrm{W}\right)$, altitude entre 6-10 $\mathrm{m}$ acima do nível do mar. A mata ciliar desta área não é contínua, são observados fragmentos ao longo do rio. O local escolhido abrange uma fração de mata em boas condições da região. A mata da margem direita do rio é mais alta, depois ocorrem áreas inundáveis, banhados e, mais distantes, campos de pastagens. Na margem esquerda, a mata foi devastada para instalação de um balneário (DANIEL, 1991). Mata ciliar do rio Maquiné, em um fragmento de mata ciliar às margens do rio Maquiné (2940'47.99”'S, 50¹1'20.03”W), no município de Maquiné, com área aproximada de 622 $\mathrm{km}^{2}$, inserido nas encostas da Serra Geral na Planície Costeira. A região compõe a Reserva da Biosfera da Mata Atlântica reconhecida pela UNESCO, desde 1992 e a Reserva Biológica da Serra Geral, estando ambas dentro do domínio da Mata Atlântica e ecossistemas associados. Essa região é considerada de extrema importância à conservação da Mata Atlântica brasileira (CONSERVATION INTERNATIONAL DO BRASIL et al., 2000), provida de uma grande biodiversidade e fragilidade em relação à antropização.

Amostragens. Foram realizadas saídas de campo durante dois anos (01 de agosto de 2007 a 06 de junho de 2009), sendo efetuadas duas coletas por estação em cada rio, totalizando 64 ao final do trabalho. Para avaliar a diversidade da fauna de aranhas foram estabelecidas seis transecções em cada mata, sendo cada transecção uma linha reta sem exceder mais do que dois metros em cada lateral. Em cada rio foram utilizados três grupos de duas transecções em diferentes pontos do local (microambientes), com objetivo de elucidar aspectos da heterogeneidade espacial da área. Duas transecções foram colocadas o mais próximo possível da borda da mata com o rio (BR); outras duas foram estabelecidas no interior da mata (IM) e as duas últimas na borda oposta ao rio, entre a mata e os campos adjacentes (BC). No total, 24 transecções foram percorridas por coleta entre todas as áreas. Em cada transecção, num intervalo de 45 minutos, foram coletadas as aranhas do estrato arbóreo-arbustivo com guarda-chuva entomológico $(70 \mathrm{~cm} \mathrm{x} 70 \mathrm{~cm})$, totalizando 288 horas de coleta ao final do trabalho.

\section{Phoroncidia piratini sp. nov. (Figs 1-9, 16)}

Tipos. Holótipo $\sigma^{\top}$, mata ciliar do rio Piratini ( $31^{\circ} 54^{\prime} 06.47^{\prime}$ 'S, 52 $39^{\prime}$ '08.29'”W), Arroio Grande, Rio Grande do Sul, Brasil, 16.XI.2007, E. N. L. Rodrigues col. (MCN 47148). Parátipos: \& (MCN 47149); ○’ ㅇ (MCN 47150); 우 (MCN 47151) todos com mesma localidade, data e coletor do holótipo; $0^{7}$, 20.XI.2007 (MCN 47152); ㅇ, 7.VIII.2008 (MCN 47153); 2 o', 19.XII.2008 (MCN 47154); ㅇ, 14.II.2009 (MCN 47155); $0^{7}$ 18.IV.2009 (MCN 47156), todos com mesma localidade e coletor do holótipo; o',, , Sede Campestre do Colégio Farroupilha, Viamão, Rio Grande do Sul, Brasil, 08.X.1997, M. A. L. Marques col. (MCN 28567); ․, Estação Ecológica do Taim, Rio Grande, Rio Grande do Sul, Brasil, 17.XII.1985, A. A. Lise col., coletado com guarda-chuva entomológico (MCN 14162); 3 ơ, Estação Ecológica do Taim, Rio Grande, Rio Grande do Sul, Brasil, 17.XII.1985, M. A. L. Marques col., coletado com guarda-chuva entomológico (MCN 16100); O’, Passo dos Bugres, São Francisco de Paula, Rio Grande do Sul, Brasil, 04.XI.1998, A. L. H. Silva col. (MCN 30298).

Etimologia. O substantivo em aposição refere-se à localidade-tipo.

Diagnose. O palpo do macho de Phoroncidia piratini sp. nov. é similar ao de P. moyobamba Levi, 1964 (ver Buckup \& MARQues, 1989: figs 10, 11) pelo êmbolo alargado ectalmente e com ápice sob o condutor, mas difere pelo condutor longo, ultrapassando o vértice do címbio (Figs 1-3) e ducto no tégulo formando somente um alça (Fig. 1); abdome com pequeno tubérculo anterior (Fig. 6). A fêmea assemelha-se a Phoroncidia moyobamba (ver BucKuP \& MARQues, 1989: figs 4-8) pelo epígino ventralmente com duas aberturas circulares, difere pela distância entre as mesmas da margem posterior do epígino, dispostas em uma depressão (Fig. 4), espermatecas, em vista dorsal, próximas entre si e ductos de fertilização curtos (Fig. 5) e pela presença de tubérculos no abdome (Figs 8, 9).

Descrição. Holótipo ơ. Carapaça parcialmente encoberta pelo abdome, pouco pilosa, castanho-escura, principalmente nas margens, castanho-clara na porção mediana, afilada anteriormente, região ocular elevada. Fila ocular anterior e posterior procurva em vista dorsal (Fig. 6). Olhos com margens negras, laterais sobre tubérculos e OMA projetados. Clípeo alto (Fig. 7), rugoso. Quelíceras, enditos e coxas castanho-amareladas. Esterno rugoso e piloso, amarelo, com margens castanhas. Pernas castanho-amareladas. Abdome quadrangular mais longo do que largo, com projeção anterior sobre a carapaça (Fig. 6), com fortes pontuações e sete pares de placas esclerotinizadas de diferentes tamanhos, dorsalmente castanho, com laterais e lista longitudinal mais escuras; área mediana com pigmento branco. Lateralmente com pregas longitudinais mais escuras e filas de placas (Fig. 7), com mancha branca disposta anteriormente. Ventre com inúmeras placas de diferentes tamanhos, bastante esclerotinizadas e pontuações; com mancha branca transversal disposta no meio do abdome se estendendo pelas laterais do anel esclerotinizado que circunda as fiandeiras. Palpo com apófise tegular de Theridiidae pouco esclerotinizada com ápice lamelado sobre êmbolo (Figs 1,2), condutor largo e escavado (Figs 1-3) e ducto no tégulo formando uma alça (Fig. 1).

Medidas. Comprimento total 2,05. Carapaça: comprimento 0,77, largura 0,85, altura 0,57. Esterno: comprimento 0,47, largura 0,47. Clípeo: altura 0,40. Abdome: comprimento 1,55, largura 1,55, altura 1,25. Fórmula das pernas $4 / 1 / 2 / 3$. Comprimento I/II/III/IV: fêmures $0,53 / 0,47 / 0,42 / 0,62$; patelas $0,26 / 0,25 / 0,22 / 0,27$; tíbias $0,33 / 0,30 / 0,25 / 0,43$; metatarsos $0,31 / 0,25 / 0,23 / 0,32$; $\operatorname{tarsos} 0,26 / 0,22 / 0,26 / 0,30$. Total 1,69/1,49/1,38/1,94. Olhos, diâmetros: OMA 0,08, OLA 0,07, OMP 0,07, OLP 0,06. Interdistâncias: OMA-OMA 0,05, OMA-OLA 0,12, OMPOMP 0,08 , OMP-OLP 0,10 .

Parátipo $\odot$ (MCN 47149, Arroio Grande, RS). Carapaça parcialmente encoberta pelo abdome (Figs 8, 9), pilosa, castanho-escura nas margens, castanho-clara 

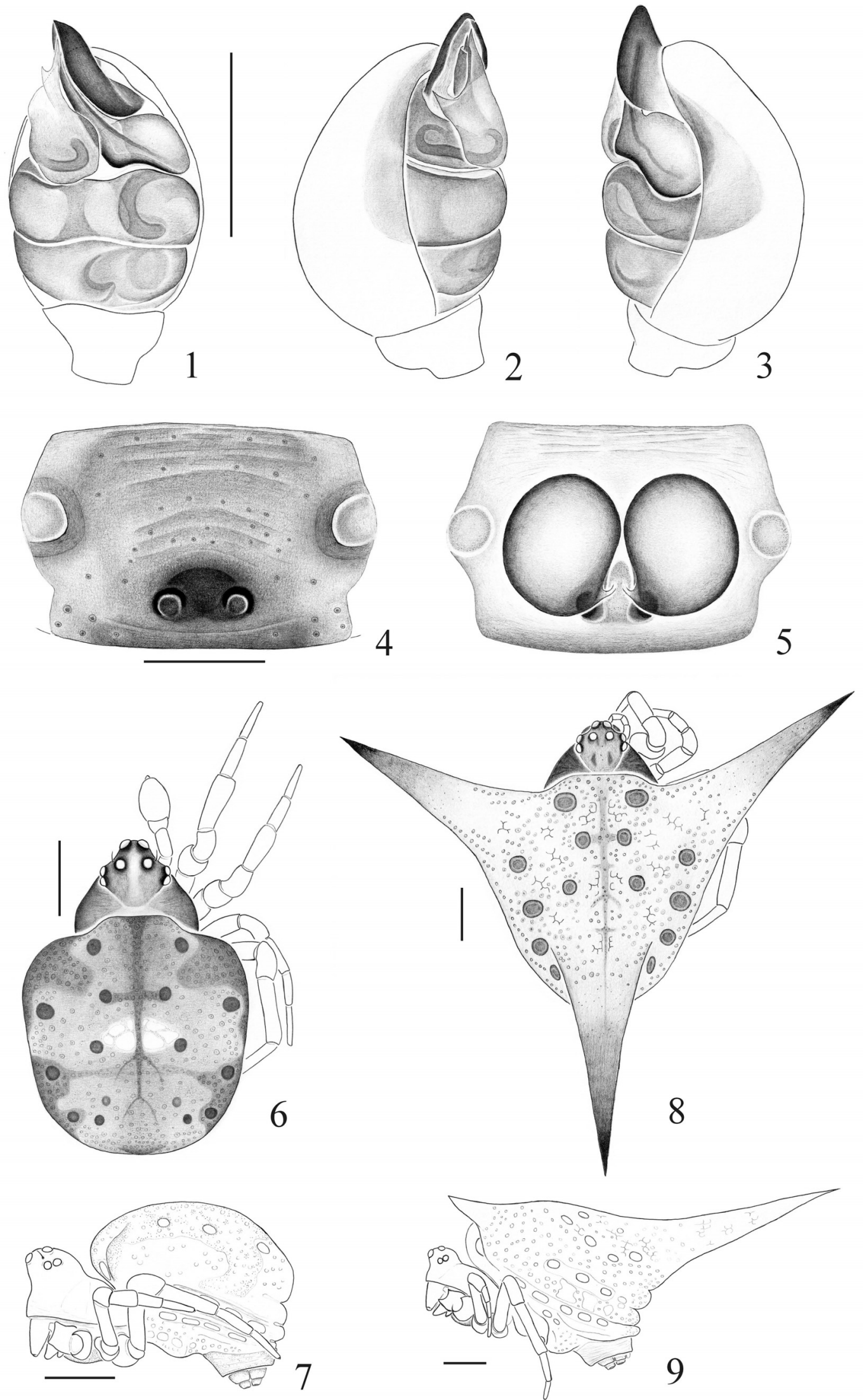

Figuras 1-9. Phoroncidia piratini sp. nov. 1-3, palpo do macho: 1, ventral; 2, mesal; 3, ectal. 4, 5, epígino: 4, ventral; 5, dorsal, clarificado. 6, 7, corpo do macho: 6, dorsal; 7, lateral. 8, 9, corpo da fêmea: 8, dorsal; 9, lateral. Barras: Figs 1-5, 0,25 mm; Figs 6-9, $0,5 \mathrm{~mm}$. 
na porção cefálica; afilada anteriormente, região ocular pouco elevada (Fig. 9). Fila ocular anterior e posterior procurva em vista dorsal. Olhos com margens negras, laterais sobre tubérculos. Clípeo, bastante piloso. Quelíceras amarelas. Enditos castanhos, porção próxima ao lábio esbranquiçada. Esterno rugoso e piloso, amarelo, com margens castanhas. Pernas amareladas, com exceção do fêmur e patela da perna IV castanho-escuros. Abdome amarelo, com dois longos tubérculos anteriores e um posterior com porções terminais castanho-avermelhadas, dorso com pequenas pontuações mais escuras, e sete pares de placas esclerotinizadas de diferentes tamanhos. Lateralmente com pregas e placas, com mancha castanhaescura entre as pregas. Ventre com inúmeras pontuações e placas de diferentes tamanhos, bastante esclerotinizadas assim como a área epigástrica, pulmonar e anel que circunda as fiandeiras. Epígino bastante esclerotinizado, com muitas cerdas, principalmente próximo a margem posterior. Na lateral da placa epiginal dois anéis esclerotinizados, duas aberturas posteriores com margens enegrecidas em uma depressão denegrida (Fig. 4); espermatecas próximas entre si e ductos de fertilização curtos na porção posterior (Fig. 5).

Medidas. Comprimento total 4,75. Carapaça: comprimento 1,25, largura 1,25, altura 0,65. Esterno: comprimento 0,70, largura 0,70. Clípeo: altura 0,35. Abdome: comprimento 4,50, largura 5,55, altura 2,35. Fórmula das pernas 4/1/2/3. Comprimento I/II/III/IV: fêmures $0,87 / 0,80 / 0,72 / 1,17$; patelas $0,37 / 0,32 / 0,32 / 0,47$; tíbias 0,60/0,44/0,44/0,77; metatarsos 0,55/0,40/0,40/0,67; tarsos 0,40/0,40/0,42/0,47. Total 2,79/2,36/2,30/3,55. Olhos, diâmetros: OMA 0,11, OLA 0,10, OMP 0,08, OLP 0,10. Interdistâncias: OMA-OMA 0,06, OMA-OLA 0,07, OMPOMP 0,10, OMP-OLP 0,11.

Variação. Comprimento total do fêmur I dos machos $(n=10)$ variou de 0,52 a 0,60 e das fêmeas $(n=10)$ variou de 0,75 a 0,95 . Em alguns espécimes, o abdome do macho apresenta-se com tonalidade mais escura, sem pigmento branco no centro e carapaça toda castanho-escura; na fêmea, alguns indivíduos com o cefalotórax todo castanho-escuro.

Distribuição. Sul do Brasil (estado do Rio Grande do Sul)(Fig. 16).

Material examinado. BRASIL, Rio Grande do Sul: Cambará do Sul, 2 ㅇ, 25.XI.1993, E. H. Buckup col. (MCN 24236); đ', 26.XI.1993, E. H. Buckup col. (MCN 24442); São Francisco de Paula, , 01-04.II.1999, L. A. Moura col. (MCN 30824); (Barragem dos Bugres), ․ 25.XI.1998, L. A. Moura col. (MCN 30957); Viamão, ㅇ, 03.VI.1956, L. Buckup col. (MCN 1625); Porto Alegre (Reserva Biológica do Lami), ơ, ㅇ, II.2000, L. E. C Schmidt col. (MCN 37947); Barra do Ribeiro (Fazenda Boa Vista), + , 16.XII.2003, Equipe Probio col., coletada com guarda-chuva entomológico (MCN 36736); Santa Maria (Perau Velho), + , 19.XI.1998, C. Kotzian \& L. Indrusiak col. (MCN 32475); Pelotas, 3 +, 26.IX.1975, A. A. Lise col. (MCN 3908); (Passo da Michaela), 9, 04.V.1996, L. A. Moura col. (MCN 27658); 2 9, 01-04.VI.1998, L. A. Moura col. (MCN 29453); Rio Grande (Estação Ecológica do Taim), , 15.X.1985, M. A. L. Marques col. (MCN 13544); 3 ㅇ, 28.XI.1985, M. Rosenau col. (MCN 13906); ㅇ, 28.XI.1985, M. A. L. Marques col. (MCN 13918); 2 9, 14.XII.1985, A. A. Lise col. (MCN 14146); 3 +, 12.XII.1985, A. A. Lise col. (MCN 14156); ठ 10.IV.1986, M. A. L. Marques col. (MCN 14639); 2 ㅇ, 10.IV.1986, E. H. Buckup col. (MCN 14712); 9 , 08.IV.1986, M. A. L. Marques col. (MCN 14731); 2 9, 09.IV.1986, M. A. L. Marques col. (MCN 14759); ㅇ, 09.IV.1986, E. H. Buckup col.
(MCN 14790); 3 9, 02.IX.1986, M. A. L. Marques col. (MCN 15783); 3 o', 02.IX.1986, A. A. Lise col. (MCN 15789); 2 우, 02.IX.1986, M. Rosenau col. (MCN 15799); ㅇ, 01.IX.1986, H. A. Gastal col. (MCN 15812); 2 9, 01.IX.1986, M. Rosenau col (MCN 15814); ㅇ, 01.IX.1986, A. A. Lise col. (MCN 15821); 02.IX.1986, E. H. Buckup col. (MCN 15844); O’, 04.XI.1986, E. H. Buckup col. (MCN 15972); 3 ㅇ, 04.XII.1986, M. A. L. Marques col. (MCN 16099); 2 ㅇ, 04.XII.1986, A. D. Brescovit col. (MCN 16113); 3 o', 04.XII.1986, A. D. Brescovit col. (MCN 16114); + , 04.XII.1986, A. D. Brescovit col. (MCN 16115); 6 q, 04.XII.1986, M. Rosenau col. (MCN 16163); O’, 04.XII.1986, E. H. Buckup col. (MCN 16179); 3 \&, 04.XII.1986, E. H. Buckup col. (MCN 16180); ㅇ, 02.XII.1986, M. Rosenau col. (MCN 16275); ㅇ, 04.XII.1986, M. C. Moraes col. (MCN 16360); 5 우, 07.I.1987, A. A. Lise col. (MCN 16624), todas da Estação Ecológica do Taim coletadas com guarda-chuva entomológico.

\section{Phoroncidia reimoseri Levi, 1964}

(Figs 10-15, 17)

Phoroncidia reimoseri LEvI, 1964:83, figs 77-81, holótipo fêmea do Rio Grande do Sul, Brasil, 1908, E. Reimoseri col., depositado no Museum of Comparative Zoology (não-examinado); Platnick, 2010

Diagnose. O palpo do macho de Phoroncidia reimoseri Levi, 1964 é similar ao de Phoroncidia nicoleti Levi, 1964 (ver Levi, 1964, fig. 24) pelo êmbolo que se estende desde sua origem ectal até o ápice do condutor que ultrapassa a margem apical do címbio, mas difere desta e das demais espécies do gênero pelo longo condutor, êmbolo e apófise tegular de Theridiidae (Figs 10-13).

Descrição ơ (MCN 47159, Maquiné, RS). Carapaça castanho-escura, pouco pilosa, parcialmente encoberta pelo abdome, afilada anteriormente, região ocular elevada. Fila ocular anterior e posterior procurva em vista dorsal (Fig. 14). Olhos com margens negras, médios projetados e laterais sobre tubérculos. Clípeo alto (Fig. 15), granuloso. Quelíceras castanho-avermelhadas. Enditos castanhos com margens amareladas. Esterno granuloso, castanho, margens enegrecidas. Pernas castanhas, exceto tíbia, metatarso e tarso da perna IV mais claros. Abdome esférico (Fig. 14), castanho-escuro com duas manchas despigmentadas posteriormente, com fortes pontuações no dorso, com placas esclerotinizadas de diferentes tamanhos. Ventre com pregas laterais e com diversas placas esclerotinizadas. Fiandeiras castanhas, no centro de um anel esclerotinizado castanho-escuro. Palpo com condutor com porção distal mais escura, apófise tegular de Theridiidae com porção distal em forma de um gancho (Figs 10-13).

Medidas. Comprimento total 1,87. Carapaça: comprimento 0,75 , largura 0,80, altura 0,62. Esterno: comprimento 0,47 , largura 0,47 . Clípeo: altura 0,47 . Abdome: comprimento 1,37, largura 1,40, altura 1,00. Fórmula das pernas 4/1/2/3. Comprimento I/II/III/IV: fêmures $0,62 / 0,47 / 0,40 / 0,65$; patelas $0,27 / 0,25 / 0,20 / 0,25$; tíbias $0,40 / 0,27 / 0,25 / 0,44$; metatarsos $0,30 / 0,22 / 0,22 / 0,32$; tarsos 0,28/0,25/0,27/0,30. Total 1,87/1,46/1,34/1,96. Olhos, diâmetros: OMA 0,08, OLA 0,07, OMP 0,08, OLP 0,06 Interdistâncias: OMA-OMA 0,08, OMA-OLA 0,21, OMPOMP 0,08, OMP-OLP 0,12.

Fêmea. Ver descrição em LeVI (1964: 83, figs 77-81).

Variação. Comprimento total do fêmur I dos machos $(n=10)$ variou de 0,57 a 0,67 . O comprimento total do fêmur 
I das fêmeas $(n=10)$ variou de 0,92 a 1,05. Em alguns espécimes, o abdome do macho se apresenta com tonalidade mais clara, assim como a carapaça tornando as duas machas despigmentadas posteriores menos evidentes.

Distribuição. Brasil (estados de São Paulo, Paraná, Santa Catarina e Rio Grande do Sul) (Fig.17).

Material examinado. BRASIL, Paraná: Foz do Iguaçu (Parque Nacional de Foz do Iguaçu, 25³6'S, 5425' W), ㅇ, 0312.II.2002, Equipe Biota col. (MCN 40806). Santa Catarina: Blumenau (Parque Natural Municipal das Nascentes do Ribeirão Garcia, 2701'S, 4909'W), +, 21-28.I.2003, Equipe Biota col. (MCN 41116); Paulo Lopes (Parque Estadual da Serra do Tabuleiro), ơ, ㅇ, 10-20.I.2003, Equipe Biota col. (MCN 42926, 42927); Rancho Queimado, o', 08-12.X.1994, L. A. Moura col. (MCN 26620); Seara (Estrada Concórdia, 27¹1'37' 'S, 52¹0'06'’W), +, 30.I.1996, A. B. Bonaldo col. (MCN 27257). Rio Grande do Sul: Iraí, 5 +, 19.XI.1975, A. A. Lise col. (MCN 3064); 12 ㅇ,
22.XI.1975, A. A. Lise col. (MCN 3130); Derrubadas (Parque Estadual do Turvo), 4 ơ , 8 क, 27-31.X.2003, R. Ott et al. col., todos coletados com guarda-chuva entomológico (MCN 37740, 37788, 37798, 38099); ơ, ㅇ, 19-22.X.2004, R. Ott et al. col. (MCN 38517); 3 ㅇ, 25-30.IV.2005, R. Ott et al. col. (MCN 39275); (Parque Estadual do Turvo, 2700' a $27^{\circ} 20^{\prime} \mathrm{S}, 53^{\circ} 40^{\prime}$ a $\left.54^{\circ} 10^{\prime} \mathrm{W}\right), 3$ \%, 11-18.I.2002, Equipe Biota col. (MCN 42870); Tenente Portela, 9 , 15-16.I.1985, A. A. Lise col. (MCN 12859, 12969); Nonoai (Parque Estadual de Nonoai), 3 \&, 13.I.1985, A. A. Lise col. (MCN 12993); Estrela Velha (Barragem de Itaúba), ơ, ㅇ, 27.XI.1999, A. B. Bonaldo col. (MCN 31638); Salto do Jacuí (Horto da CEEE), ㅇ, 19.X.1998, A. B. Bonaldo col. (MCN 30905); (Barragem de Itaúba), 2 ㅇ, 25.XI.1999, S. D. Hahn col. (MCN 33397); Torres (Parque Estadual de Itapeva), 2 , 10 14.I.2005, R. Ott et al. col. (MCN 38632); Caxias do Sul (Vila Oliva), , 15.I.1974, F. R. Meyer col. (MCN 2002); ㅇ, 04.IV.1975, A. A. Lise col. (MCN 2875); Canela, ㅇ, 02.VIII.1965, A. A. Lise col. (MCN 34); Arroio do Meio (São Jacó), 3 \&, 09.I.1985, A. A. Lise col. (MCN 12940); (Linha Alegre), , 09.I.1985, A. A. Lise col. (MCN 12953); São Francisco de Paula (Rincão dos Kroeff, $840 \mathrm{~m}$ de altitude), , 05.I.1985, A. A. Lise col. (MCN 12729);
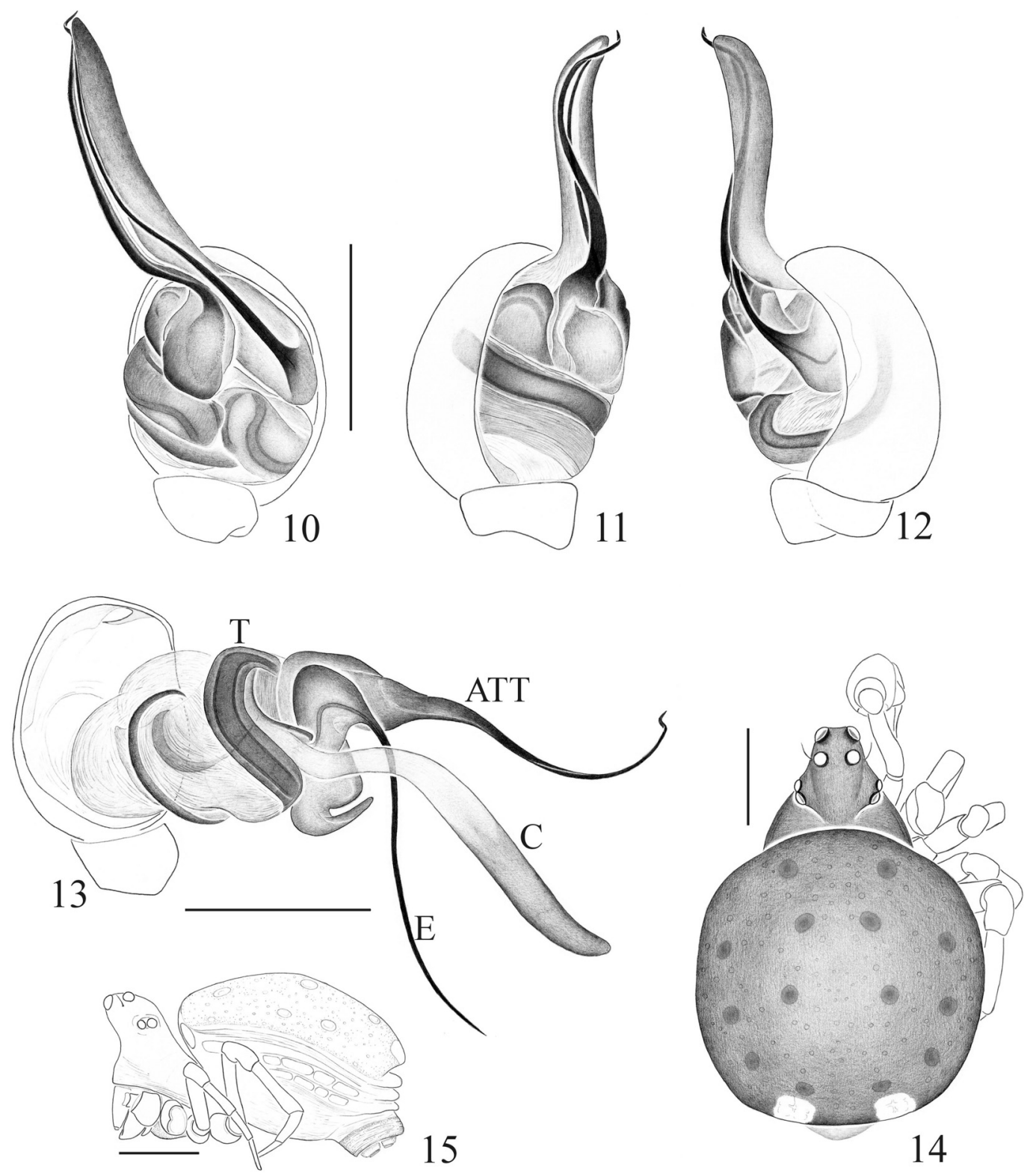

Figuras 10-15. Phoroncidia reimoseri Levi, 1964, macho: 10-13, palpo: 10, ventral; 11, mesal; 12, ectal; 13, expandido; 14, 15, corpo: 14, dorsal; 15, lateral (ATT, apófise tegular de Theridiidae; C, condutor; E, êmbolo; T, tégulo). Barras: Figs 10-13, 0,25 mm; Figs 14, 15, $0,5 \mathrm{~mm}$. 

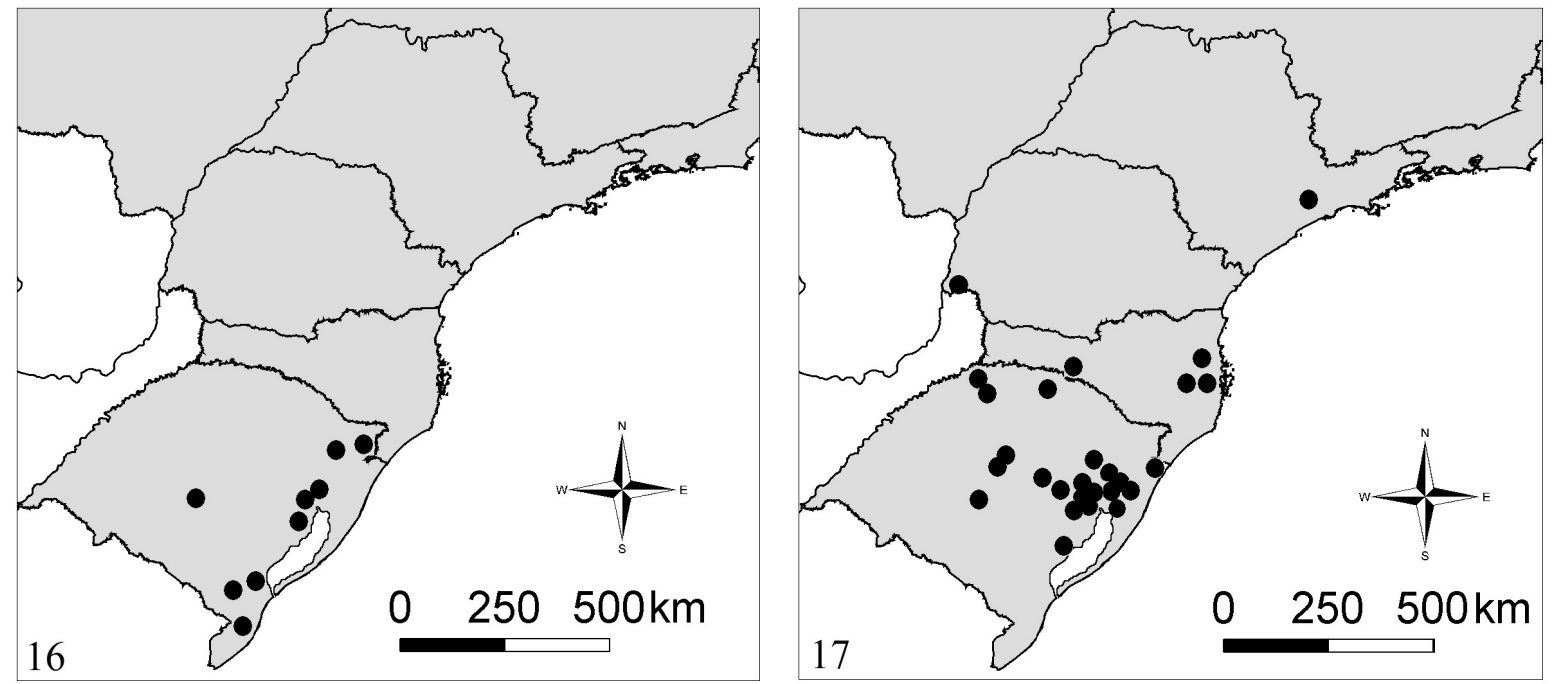

Figuras 16, 17. Mapa da distribuição das espécies estudadas: 16, distribuição de Phoroncidia piratini sp. nov.; 17, distribuição de Phoroncidia reimoseri Levi, 1964.

(Passo dos Bugres), , , 06.XI.1998, A. B. Bonaldo col. (MCN 30048); 3 9 , 01-04.II.1999, A. B. Bonaldo col. (MCN 30823); (Barragem dos Bugres), ơ, ㅇ, 25.XI.1998, L. A. Moura col. (MCN 30956, 30984); Maquiné (Reserva da FEPAGRO) $\left(29^{\circ} 39^{\prime} \mathrm{S}, 50^{\circ} 12^{\prime} \mathrm{W}\right)$, 웅, 18-27.I.2002, Equipe Biota col. (MCN 43212); (mata ciliar do rio

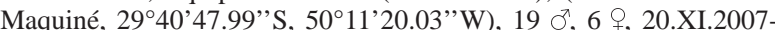
27.IV.2009, E. N. L. Rodrigues col., todos coletados com guardachuva entomológico (MCN 47159, 47161, 47164-47170); Parobé (mata ciliar do rio dos Sinos, 29 41'06.94"'S, 5051'05.98"W), 42 O', 60 ค, 19.IX.2007-25.IV.2009, E. N. L. Rodrigues col., todos coletados com guarda-chuva entomológico (MCN 47157, 47158, 47160, 47163, 47174-47180); Santa Maria (Reserva Biológica do Ibicuí-Mirim), ơ, 19.XII.1991, N. Silveira col. (MCN 21878); (Lar Metodista), 2 , 12.VII.2004, L. Indrusiak col. (MCN 37862); Campo Bom, 3 ㅇ, 01.IV.1985, C. J. Becker col. (MCN 13258); ㅇ, 14.XII.1987, C. J. Becker col. (MCN 19505); Novo Hamburgo, $q$, 20.X.1986, C. J. Becker col. (MCN 19511); São Leopoldo (Banhado Steigleder), 2 +, 04.IV.1976, C. J. Becker col. (MCN 3637); (Rio dos Sinos), +, 06.VIII.1982, C. J. Becker col. (MCN 10666); ㅇ, 21.III.1983, C. J. Becker col. (MCN 11484); 우, 06.V.1983, C. J. Becker col. (MCN 11638); Montenegro,, , 11.VIII.1977, E. H. Buckup col. (MCN 6261); 6 , 06.X.1977, A A. Lise col. (MCN 6765, 6767); 18 , 06.X.1977, H. Bischoff col. (MCN 6766, 6812); O’, 06.X.1977, E. H. Buckup col. (MCN 6814); O’, 17.XI.1977, E. H. Buckup col. (MCN 7237); 7 क, 17.XI.1977, A. A. Lise col. (MCN 7238); 2 O, 3 9, 01.XII.1977, A. A. Lise col. (MCN 7422, 7427, 7477); 10 ㅇ, 20.XII.1977, A. A. Lise col. (MCN 7657); Triunfo, 2 क, 20.X.1977, A. A. Lise col. (MCN 6926); ㅇ, 12.V.1981, E. H. Buckup col. (MCN 9671); Viamão, ㅇ, 01.XI.1961, C. A. Hartlieb col. (MCN 1281); (Parque Saint Hilaire), 2 +, 30.IV.1976, A. A. Lise col. (MCN 4180); Porto Alegre (Morro Santana), +, 15.XII.1989, A. A. Lise col. (MCN 19170); Cristal (mata ciliar do rio Camaquã, $31^{\circ} 01$ '01.70"'S, 51 ${ }^{\circ} 56$ '42.01"W), 2 o', 2 ?, 26.VII.2007-12.VI.2009, E. N. L. Rodrigues col., todos coletados com guarda-chuva entomológico (MCN 47162, 4717147173).

Ecologia. Nas matas ciliares estudadas, a família Theridiidae foi a mais abundante e rica em espécies, totalizando 4363 aranhas adultas em 102 espécies. Phoroncidia piratini, pertencente a esta família, foi exclusiva do rio Piratini, não foi uma espécie muito abundante, sendo coletados 11 indivíduos, com predomínio de machos sobre fêmeas (razão sexual 1,2:1). Phoroncidia reimoseri foi bem mais abundante, registrando 139 indivíduos, diferente da espécie anterior, os machos foram menos abundantes que as fêmeas $(1: 1,17)$. Esta espécie foi registrada em todas as matas ciliares, com exceção do rio Piratini, predominando na mata ciliar do rio dos Sinos ( $\mathrm{n}=106)$.

Phoroncidia reimoseri também foi registrada por PoDgaiski et al. (2004) no Parque Estadual do Turvo, em Derrubadas, noroeste do Rio Grande do Sul, área que protege o único remanescente representativo e preservado da Floresta do Alto Uruguai no Brasil INDRUSIAK \& KOTZIAN (1998) registraram esta espécie para Santa Maria, sendo um dos locais estudados uma mata de galeria.

No padrão de distribuição ao longo das coletas, somente na primavera foram amostrados juntos machos e fêmeas de Phoroncidia piratini (Fig. 18). A espécie Phoroncidia reimoseri também apresentou os maiores picos na primavera, principalmente de machos (Fig. 19). Possivelmente na primavera ocorre a época de reprodução das espécies. Os machos, de ambas as espécies, não foram coletados no inverno e outono, única exceção um macho de $P$. piratini no outono do $2^{\circ}$ ano de coleta. No inventário de Podgaiski et al. (2004) Phoroncidia reimoseri seguiu o mesmo padrão predominando na primavera.

Além de um microclima específico, as aranhas para o local da construção da teia, necessitam também de certa demanda espacial (Foelix, 1996), entretanto, as duas espécies aqui estudadas demonstram padrões diferentes de escolha do microambiente (Fig. 20). Phoroncidia piratini, no estrato arbóreo-arbustivo aqui estudado, apresentou preferência pela borda do campo, já $P$. reimoseri tem ampla predileção pelo interior da mata para construção de suas teias e local de sua permanência e foi menos abundante na borda do campo.

Aranhas deste gênero são conhecidas por construírem teias simples, chegando ao extremo de somente um fio de seda adesivo (Marples, 1955; EBERHARD, 1981), provavelmente, são aranhas 

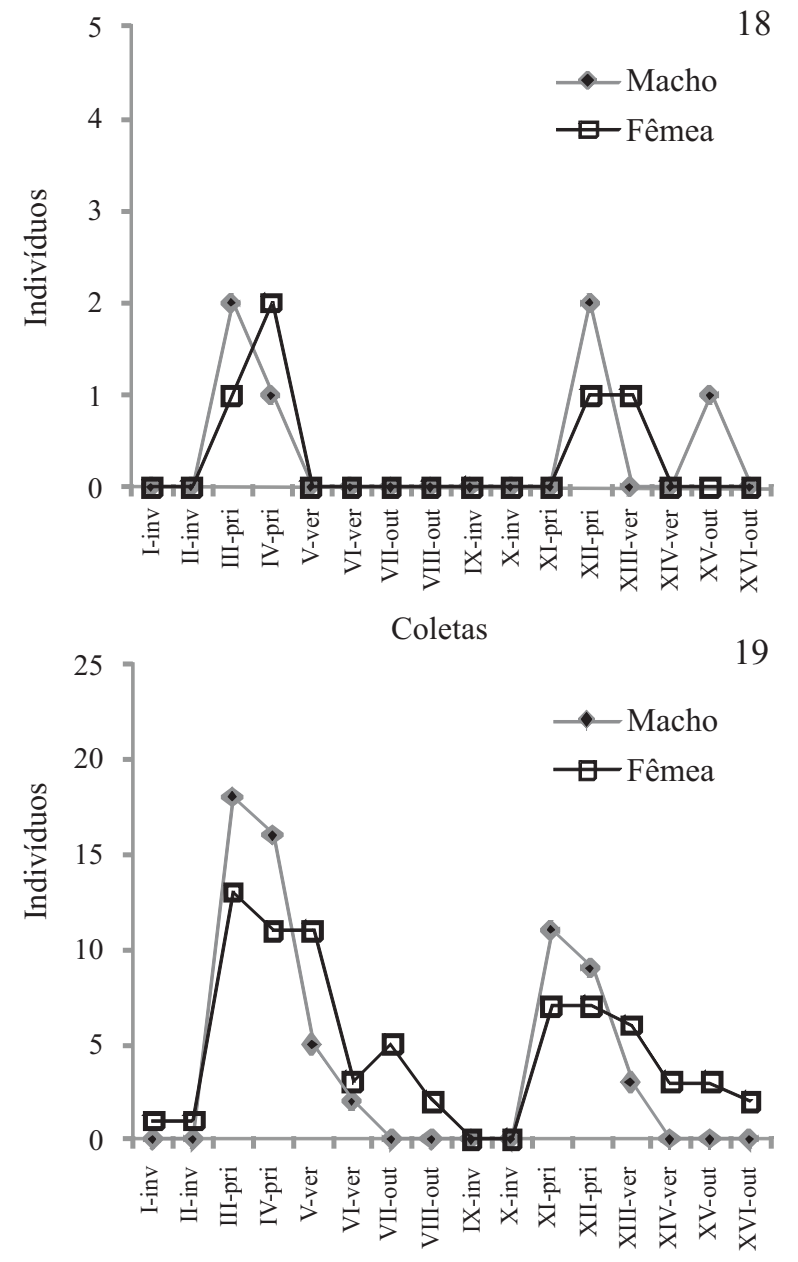

Coletas

Figuras 18, 19. Fenologia das espécies de Phoroncidia coletadas entre 01 de agosto de 2007 a 06 de junho de 2009 em matas ciliares no sul do Brasil: 18, Phoroncidia piratini sp. nov.; 19, Phoroncidia reimoseri Levi, 1964 (inv, inverno; pri, primavera; ver, verão; out, outono).

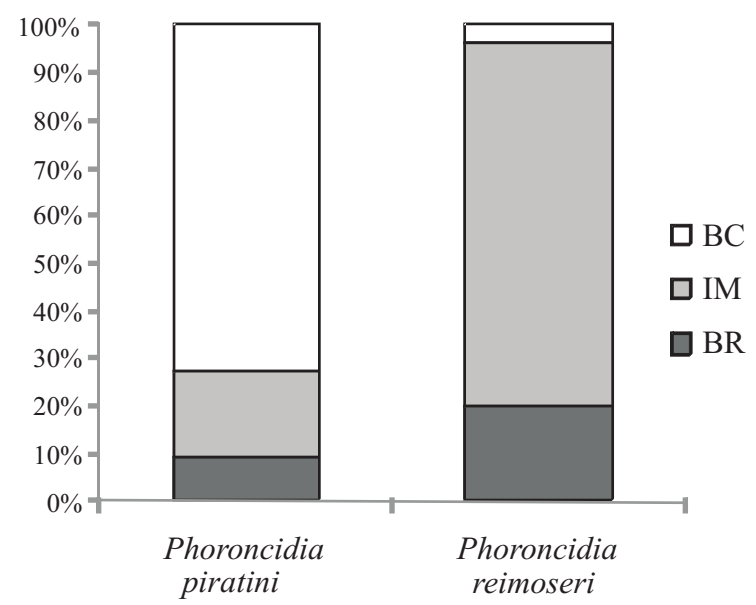

Figura 20. Preferência das espécies de Phoroncidia por microambientes em matas ciliares no sul do Brasil, coletadas entre 01 de agosto de 2007 a 06 de junho de 2009 (BC, borda do campo; IM, interior da mata; $\mathrm{BR}$, borda do rio). especialistas pelas características das teias. Especializações por tipos de presas podem levar a uma preferência por um determinado hábitat (UETZ, 1977), no caso das aranhas aqui estudadas, possivelmente, tem uma maior dependência por presas. Devido ao pouco conhecimento sobre a história natural das espécies deste gênero (LEVI, 1964), os dados aqui apresentados contribuem para o melhor conhecimento da ecologia das espécies em matas ciliares.

Agradecimentos. À curadora da coleção araneológica do MCN Erica H. Buckup, pelo acesso ao material e à bibliografia. À Patrícia E. S. Rodrigues (MCN) pelo auxílio nas coletas. Ao CNPq pela bolsa de doutorado concedida a E. N. L. Rodrigues pelo Programa de Pós-Graduação em Biologia Animal da Universidade Federal do Rio Grande do Sul (processo 140586/2007-5) e ao professor Milton de Souza Mendonça, Jr. (UFRGS) pela orientação. Ao projeto BIOTA/FAPESP - Programa Virtual de Biodiversidade (www.biotasp.org.br) - pela doação de material.

\section{REFERÊNCIAS BIBLIOGRÁFICAS}

Agnarsson, I.; Coddington, J. A. \& Knoflach, B. 2007. Morphology and evolution of cobweb spider male genitalia (Araneae, Theridiidae). Journal of Arachnology 35:334-395.

Buckup, E. H. \& Marques, M. A. L. 1989. Aranhas Theridiidae da Ilha de Maracá, Roraima, Brasil. I. Nova espécie de Echinotheridion e descrição da fêmea de Phoroncidia moyobamba (Araneae). Iheringia, Série Zoologia, 69:123129.

Conservation International do BRasil; Fundação SOS Mata AtLÂNTICA; FundaÇão Biodiversitas; Instituto de Pesquisas ECológicas; SeCRETARIA do Meio Ambientes do Estado de São Paulo \& SEMAD/Instituto Estadual de Florestas, MG. 2000. Avaliações e ações prioritárias para a conservação da biodiversidade da Mata Atlântica e Campos Sulinos. Brasília, Ministério do Meio Ambiente/SBF. 40p.

Daniel, A. 1991. Estudo fitossociológico arbóreo/arbustivo da mata ripária da Bacia Hidrográfica do rio dos Sinos, RS. Pesquisas, Botânica, 42:1-199.

Eberhard, W. G. 1981. The single line web of Phoroncidia studo Levi (Araneae: Theridiidae): a prey attract ant? Journal of Arachnology 9:229-232.

FoElix, R. F. 1996. Biology of spiders. New York, Oxford University. 336p.

IndRusiaK, L. F. \& KotZian, C. B. 1998. Inventário das aranhas arborícolas de três regiões de Santa Maria, RS, Brasil. Revista Ciência e Natura 20:187-214.

Levi, H. W. 1964. American spiders of the genus Phoroncidia (Araneae: Theridiidae). Bulletin of the Museum of Comparative Zoology 131(3):65-86.

Levi, H. W. \& Levi, L. R. 1962. The genera of the family Theridiidae. Bulletin of the Museum of Comparative Zoology 127(1):1-71.

Marples, B. J. 1955. A new type of web spun by spiders of the genus Ulesanis with the description of two new species. Proceedings Zoological Society of London 125:751-760.

Platnick, N. I. 2010. The world spider catalog, version 10.5. American Museum of Natural History. Disponível em: <http:/ /research.amnh.org/entomology/spiders/catalog/index.html>. Acesso em: 16.05.2010.

Podgaiski, L. R.; Ott, R.; Rodrigues, E. N. L.; Buckup, E. H. \& Marques, M. A. L. 2007. Araneofauna (Arachnida; Araneae) do Parque Estadual do Turvo, Rio Grande do Sul, Brasil. Biota Neotropica 7(n2): http://www.biotaneotropica.org.br/v7n2/pt/ abstract?inventory+bn01107022007.

Uetz, G. W. 1977. Coexistence in a guild of wandering spiders. Journal of Animal Ecology 46:531-541.

Recebido em junho de 2010. Aceito em setembro de 2010. ISSN 0073-4721

Artigo disponível em: www.scielo.br/isz 